\section{6-Methyloxazolomycin, a New Antimicrobial and Cytotoxic Substance Produced by a Streptomyces sp.}

\section{Geonseek Ryu, Seunguin Hwang and SI-KWAN KIM*}

Korea Ginseng \& Tobacco Research Institute, 302 Shinseong-dong, Yuseong-ku, Taejon 305-345, Korea

(Received for publication August 11, 1997)

Triene antibiotics containing $\beta$-lactone- $\gamma$-lactam bicyclospiro and oxazole rings are unique metabolites produced by Streptomyces spp., such as oxazolomycins ${ }^{1,2)}$ and curromycins. ${ }^{3,4)}$ In the course of our continuing search for novel antimicrobial agents from microorganisms, an actinomycete strain Streptomyces sp. isolated from a soil sample collected in Taejon, Korea, was found to produce a new antibacterial 16-methyloxazolomycin (1). In this paper, we describe the production, isolation, structural elucidation and biological activities of $\mathbf{1}$.

The strain was cultured in the seed medium consisting of glucose $2 \%$, starch $1 \%$, soybean flour $2.5 \%$, yeast extract $0.4 \%, \mathrm{NaCl} 0.2 \%, \mathrm{~K}_{2} \mathrm{HPO}_{4} 0.005 \%$ and beef extract $0.1 \%$ (adjusted to $\mathrm{pH} 7.3$ before sterilization). The seed culture was carried out on a rotary shaker $(250 \mathrm{rpm})$ at $28^{\circ} \mathrm{C}$ for 24 hours in 500 -ml Erlenmeyer flasks containing $100 \mathrm{ml}$ of the seed medium. Then, the seed culture $(100 \mathrm{ml})$ was inoculated to a 50 -liter jar fermenter containing 10 liters of the production medium (antifoam $0.08 \%$ ). Fermentation was carried out at $27^{\circ} \mathrm{C}$ for 4 days with aeration (10 liters/minute) under constant agitation $(250 \mathrm{rpm})$.

The isolation of 16-methyloxazolomycin was guided by the use of Bacillus subtilis IAM 1069 as a test organism. The culture broth was centrifuged to separate the mycelial cake. The mycelial cake was stirred overnight in $70 \%$ aqueous acetone (5 liters) and filtered. The filtrate was concentrated in vacuo to remove the organic solvent, resulting in an aqueous solution. The combined filtrates were passed through a Diaion HP-20 (Mitsubishi Kasei, Japan) column, and washed with $\mathrm{H}_{2} \mathrm{O}$ followed by $\mathrm{MeOH}$. The methanol eluate was partitioned by the Kupchan's scheme. ${ }^{5)}$ The $\mathrm{CH}_{2} \mathrm{Cl}_{2}$ layer concentrated was fractionated by ODS flash chromatography with aqueous $\mathrm{MeOH}$. The active $80 \% \mathrm{MeOH}$ fraction was gel-filtered on Sephadex LH-20 with $n$-hexane $/ \mathrm{CH}_{2} \mathrm{Cl}_{2} / \mathrm{MeOH}$ $(4: 2: 1)$ to afford an active fraction. This fraction was finally purified by reversed-phase HPLC with $63 \%$ $\mathrm{MeOH}$ containing $0.5 \mathrm{~mm} \mathrm{NaClO}_{4}$ to yield 16-methyloxazolomycin $(1,6.0 \mathrm{mg})$ together with the known oxazolomycin $(2,15 \mathrm{mg})$.

Physico-chemical properties of $\mathbf{1}$ are as follows: pale yellow amorphous powder; $[\alpha]_{\mathrm{D}}^{23}+3.6^{\circ}(c 0.52, \mathrm{MeOH})$; UV $\lambda_{\max }(\mathrm{MeOH}) \mathrm{nm}(\varepsilon) 228(23,000), 266(27,200), 275$ $(32,000)$ and $285(27,000)$; IR $v_{\max }$ (film) $3350(\mathrm{OH}$ or $\mathrm{NH}), 2930,1825$ ( $\beta$-lactone), 1690 (amide), 1645, 1240, $1040 \mathrm{~cm}^{-1}$; ESI-MS $m / z 724(\mathrm{M}+\mathrm{MeOH}+\mathrm{Na})^{+}, 692$ $(\mathrm{M}+\mathrm{Na})^{+}, 579,507$; HRESI-MS $m / z 670.3706$ cacld. for $\mathrm{C}_{36} \mathrm{H}_{52} \mathrm{~N}_{3} \mathrm{O}_{9}(\Delta-2.6 \mathrm{mmu}) ;{ }^{1} \mathrm{H}$ NMR and ${ }^{13} \mathrm{C}$ NMR data are shown in Table 1.

The ESI mass data of 16-methyloxazolomycin suggested that 1 was 14 units larger than oxazolomycin (2). The NMR spectra of $\mathbf{1}$ were similar to those of oxazolomycin except for the appearance of a doublet methyl $(\delta 1.69 / 17.9)$ and a quartet oxymethine $(\delta$ $4.82 / 78.4$ ) instead of an oxymethylene in 2 , thus indicating that one hydrogen of the oxymethylene in 2 was displaced with the methyl as was the case with curromycin B (3). This was substantiated by interpretation of the HMBC spectrum ${ }^{6}$ (Table 1); the methyl group was placed at $\mathrm{C} 16$ by $\mathrm{HMBC}$ cross peaks between $\mathrm{H} 16$ and $\mathrm{C} 3$ and $\mathrm{C} 15$, and between $16-\mathrm{CH}_{3}$ and $\mathrm{C} 16$ and $\mathrm{C} 15$, thus constructing gross structure 1 belonging to the oxazolomycin class. Because ${ }^{1} \mathrm{H}$ NMR spectrum measured in $\mathrm{CDCl}_{3}$ revealed overlapped signals in the olefinic region, the geometries of the triene and diene systems

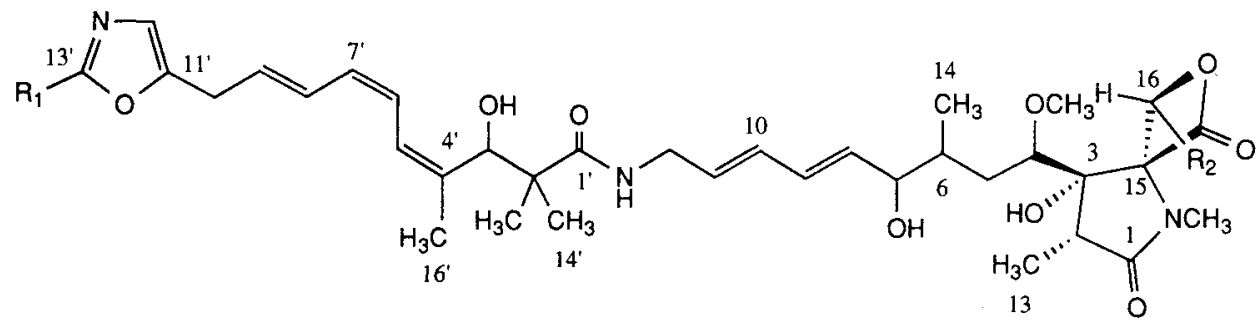


Table 1. ${ }^{1} \mathrm{H}$ and ${ }^{13} \mathrm{C}$ NMR data of 16-methyloxazolomycin in DMSO- $d_{6}$.

\begin{tabular}{|c|c|c|c|}
\hline Position & $\delta^{13} \mathrm{C}$ & $\delta{ }^{1} \mathrm{H}$ (mult., $J \mathrm{~Hz}$ ) & HMBC \\
\hline 1 & 174.2 & & $\mathrm{H} 2, \mathrm{NCH}_{3}, \mathrm{H} 13$ \\
\hline 2 & 44.7 & $2.34(\mathrm{H}, \mathrm{q}, 6.6)$ & $\mathrm{H} 13$ \\
\hline 3 & 81.8 & & $\mathrm{H} 2, \mathrm{H} 13, \mathrm{H} 16,3-\mathrm{OH}$ \\
\hline 4 & 83.7 & $3.38(\mathrm{H}, \mathrm{t}, 6.9)^{\mathrm{a}}$ & $\mathrm{OCH}_{3}, 3-\mathrm{OH}$ \\
\hline \multirow[t]{2}{*}{5} & 33.1 & $1.95(\mathrm{H}, \mathrm{m})$ & H7, H14 \\
\hline & & $1.15(\mathrm{H}, \mathrm{m})$ & \\
\hline 6 & 37.9 & $1.58(\mathrm{H}, \mathrm{m})$ & $\mathrm{H} 8, \mathrm{H} 14,7-\mathrm{OH}$ \\
\hline 7 & 76.4 & $3.81(\mathrm{H}, \mathrm{m})$ & $\mathrm{H} 8, \mathrm{H} 9,7-\mathrm{OH}$ \\
\hline 8 & 129.5 & $5.57(\mathrm{H}, \mathrm{dd}, 16.8,11.2)$ & $\mathrm{H} 9, \mathrm{H} 10,7-\mathrm{OH}$ \\
\hline 9 & 130.0 & $6.14(\mathrm{H}, \mathrm{dd}, 11.2,16.8)$ & H7, H10, H11 \\
\hline 10 & 131.5 & $6.09(\mathrm{H}, \mathrm{dd}, 14.3,10.8)$ & $\mathrm{H} 8, \mathrm{H} 11, \mathrm{H} 12$ \\
\hline 11 & 134.0 & $5.59(\mathrm{H}, \mathrm{m})$ & $\mathrm{H} 10, \mathrm{H} 12$ \\
\hline 12 & 42.2 & $3.71(2 \mathrm{H}, \mathrm{m})$ & $\mathrm{H} 10, \mathrm{H} 11, \mathrm{NH}$ \\
\hline 13 & 20.8 & $1.03(3 \mathrm{H}, \mathrm{d}, 6.6)$ & $\mathrm{H} 2$ \\
\hline 14 & 17.2 & $0.86(3 \mathrm{H}, \mathrm{d}, 6.2)$ & \\
\hline 15 & 84.7 & & $\mathrm{H} 4, \mathrm{H} 16,16-\mathrm{CH}_{3}, \mathrm{NCH}_{3}$ \\
\hline 16 & 78.4 & $4.82(\mathrm{H}, \mathrm{q}, 6.3)$ & $16-\mathrm{CH}_{3}$ \\
\hline 17 & 170.2 & & $\mathrm{H} 16$ \\
\hline $1^{\prime}$ & 176.2 & & $\mathrm{H} 12, \mathrm{H}^{\prime}, \mathrm{H} 14^{\prime}, \mathrm{H} 15^{\prime}, \mathrm{NH}$ \\
\hline $2^{\prime}$ & 46.7 & & $\mathrm{H} 14^{\prime}, \mathrm{H} 15^{\prime}, 3^{\prime}-\mathrm{OH}$ \\
\hline $3^{\prime}$ & 74.4 & $4.59(\mathrm{H}, \mathrm{s})$ & $\mathrm{H} 5^{\prime}, \mathrm{H} 14^{\prime}, \mathrm{H} 15^{\prime}, \mathrm{H} 16^{\prime}$ \\
\hline $4^{\prime}$ & 141.0 & & $\mathrm{H} 3^{\prime}, \mathrm{H} 6^{\prime}, \mathrm{H} 16^{\prime}$ \\
\hline $5^{\prime}$ & 124.6 & $6.35(\mathrm{H}, \mathrm{d}, 11.3)$ & $\mathrm{H} 3^{\prime}, \mathrm{H} 16^{\prime}$ \\
\hline $6^{\prime}$ & 125.5 & $6.27(\mathrm{H}, \mathrm{dd}, 10.9,11.3)$ & $\mathrm{H}^{\prime}, \mathrm{H} 7^{\prime}$ \\
\hline $7^{\prime}$ & 128.2 & $5.90(\mathrm{H}, \mathrm{dd}, 11.5,10.9)$ & $\mathrm{H}^{\prime}, \mathrm{H} 9^{\prime}$ \\
\hline $8^{\prime}$ & 129.1 & $6.70(\mathrm{H}, \mathrm{dd}, 11.5,14.5)$ & $\mathrm{H} 6^{\prime}, \mathrm{H} 7^{\prime}, \mathrm{H} 9^{\prime}, \mathrm{H} 10^{\prime}$ \\
\hline $9^{\prime}$ & 129.8 & $5.75(\mathrm{H}, \mathrm{dt}, 14.5,4.5)$ & $\mathrm{H}^{\prime}, \mathrm{H} 10^{\prime}$ \\
\hline $10^{\prime}$ & 29.4 & $3.52(2 \mathrm{H}, \text { brd, } 7.0)^{\mathrm{b}}$ & $\mathrm{H} 8^{\prime}, \mathrm{H} 9^{\prime}$ \\
\hline $11^{\prime}$ & 151.1 & & $\mathrm{H} 9^{\prime}, \mathrm{H} 10^{\prime}, \mathrm{H} 12^{\prime}, \mathrm{H} 13^{\prime}$ \\
\hline $12^{\prime}$ & 122.9 & $6.84(\mathrm{H}, \mathrm{s})$ & $\mathrm{H} 10^{\prime}, \mathrm{H}_{1} 3^{\prime}$ \\
\hline $13^{\prime}$ & 152.1 & $8.14(\mathrm{H}, \mathrm{s})$ & $\mathrm{H} 12^{\prime}$ \\
\hline $14^{\prime}$ & 22.6 & $0.95(3 \mathrm{H}, \mathrm{s})$ & $\mathrm{H} 3^{\prime}, \mathrm{H}^{\prime} 5^{\prime}$ \\
\hline $15^{\prime}$ & 26.1 & $1.10(3 \mathrm{H}, \mathrm{s})$ & $\mathrm{H} 3^{\prime}, \mathrm{H} 14^{\prime}$ \\
\hline $16^{\prime}$ & 20.9 & $1.70(3 \mathrm{H}, \mathrm{s})$ & $\mathbf{H} 3^{\prime}, \mathrm{H} 5^{\prime}$ \\
\hline $16-\mathrm{CH}_{3}$ & 17.9 & $1.69(3 \mathrm{H}, \mathrm{d}, 6.3)$ & H3 \\
\hline $\mathrm{NCH}_{3}$ & 27.1 & $2.77(3 \mathrm{H}, \mathrm{s})$ & \\
\hline $\mathrm{OCH}_{3}$ & 57.1 & $3.15(3 \mathrm{H}, \mathrm{s})$ & $\mathrm{H} 4$ \\
\hline $3-\mathrm{OH}$ & & $5.31(\mathrm{H}, \mathrm{s})$ & \\
\hline $7-\mathrm{OH}$ & & $4.82(\mathrm{H}, \mathrm{s})$ & \\
\hline $3^{\prime}-\mathrm{OH}$ & & $5.49(\mathrm{H}, \mathrm{s})$ & \\
\hline $\mathrm{NH}$ & & $7.63(\mathrm{H}$, br t, 5.9) & \\
\hline
\end{tabular}

a,b Proton chemical shifts and coupling constants were deduced from the ${ }^{1} \mathrm{H}$ NMR spectrum in $\mathrm{CDCl}_{3}$.

were determined by the spin coupling constants measured in DMSO- $d_{6}$ (Table 1). Furthermore, stereochemistry of $\beta$-lactone- $\gamma$-lactam spiro ring except for $\mathrm{C} 16$ was deduced to be identical with that of oxazolomycin by NOESY experiments $^{7)}$ (Fig. 1). NOESY cross peaks H16/3-OH and $16-\mathrm{CH}_{3} / \mathrm{NCH}_{3}$ exhibited $S$ configuration of $\mathrm{C} 16$.

16-Methyloxazolomycin (1) showed antibacterial and antialgal activities against Bacillus subtilis 1069 (MIC, $5.0 \mu \mathrm{g} / \mathrm{ml}$ ) and Chlorella vulgaris IFO 15941 (MIC, $10 \mu \mathrm{g} / \mathrm{ml})$, respectively and cytotoxicities $\left(\mathrm{IC}_{50}, 0.23 \mu \mathrm{g} /\right.$ $\mathrm{ml}$ against $\mathrm{P} 388$ leukemia cells; $4.6 \mu \mathrm{g} / \mathrm{ml}$ against A-549 human lung adenocarcinoma cells).
Fig. 1. Partial correlations obtained from NOESY experiment for 1.

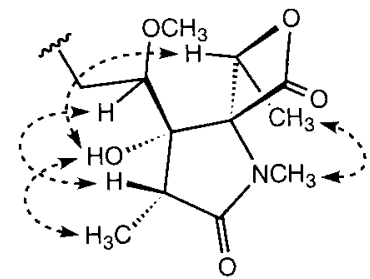




\section{References}

1) Mori, T.; K. Takahashi, M. Kashiwabara, D. Uemura, C. Katayama, S. Iwadare, Y. Shizuri, R. Mrtomo, F. NaKano \& A. Matsuzaki: Structure of oxazolomycin, a novel $\beta$-lactone antibiotic. Tetrahedron Lett. 26: $1073 \sim$ 1076,1985

2) Takahashi, K.; M. Kawabara, D. Uemura, S. Wadare, R. Mitomo, F. Nakano \& A. Matsuzaki: Structure of neooxazolomycin, an antitumor antibiotic. Tetrahedron Lett. 26: $1077 \sim 1078,1985$

3) Ogura, M.; H. Nakayama, K. Furihata, H. Seto \& N OTAKE: Structure of a new antibiotic curromycin A produced by a genetically modified strain of Streptomyces hygroscopicus, a polyether antibiotic producing organism. J. Antibiotics 38: 669 673, 1985
4) Ogura, M.; H. Nakayama, K. Furihata, A. Shimazu, H. Seto \& N. OtaKe: Isolation and structural determination of a new antibiotic curromycin B. Agric. Biol. Chem. 49: 1909 1910, 1985

5) Kupchan, S. M.; R. W. Britton, M. F. Ziegler \& C. W. Sigel: Bruceantin, a new potent antileukemic simaroubolide from Brucea antidysenterica. J. Org. Chem. 38: $178 \sim 179,1973$

6) Bax, A.; A. Azolos, Z. Dinya \& K. Sudo: Structure elucidation of the antibiotic desertomycin through the use of new two-dimensional NMR techniques. J. Am. Chem. Soc. 108: 8056 8063, 1986

7) Bodenhausen, G.; H. Kogler \& R. R. Ernst: Selection of coherence-transfer pathways in NMR pulse experiments. J. Magn. Reson. 58: 370 388, 1984 